\title{
Structure of the Blm10-20S Proteasome Complex by Single Particle Cryo- Electron Microscopy
}

Joaquin Ortega $^{1 *}$, Jack Iwanczyk ${ }^{1}$, Kianoush Sadre-Bazzaz ${ }^{2}$, Katherine Ferrell ${ }^{2}$, Elena

Kondrashkina $^{3}$, Timothy Formosa ${ }^{2}$, Christopher P. Hill ${ }^{2}$.

${ }^{1}$ Department of Biochemistry and Biomedical Sciences, McMaster University, 1200 Main Street West, Hamilton, Ontario, L8N3Z5, Canada.

${ }^{2}$ Department of Biochemistry, University of Utah School of Medicine, 15 North Medical Drive East, Salt Lake City, UT 84112-5650, USA;

${ }^{3}$ Biophysics Collaborative Access Team (BioCAT), BCPS Department, Illinois Institute of Technology, 3101

S. Dearborn, Chicago, IL 60616, USA.

The $20 \mathrm{~S}$ proteasome is regulated at multiple levels including association with endogenous activators. Two activators have been described for the yeast 20S proteasome: the 19S regulatory [1] particle and the Blm10 protein [2], which is the yeast homolog of mammalian PA200 [3].

Blm10 was initially reported to regulate proteasome maturation, and therefore it was questioned whether PA200 and Blm10 are functionally related [4]. Recent studies have shown that the sequence of Blm10 contains multiple HEAT-repeats and activates mature proteasomes, suggesting functional homology with PA200 [5]. However, the labile nature of these complexes has complicated their structural characterization.

We have developed a purification method that produces high yields of stoichiometric Blm10-mature yeast $20 \mathrm{~S}$ proteasome complexes. To determine whether Blm10 interacts with the $20 \mathrm{~S}$ in a similar fashion to its mammalian PA200 homolog, we constructed a three-dimensional (3D) model of the Blm10-20S complex from cryo-electron microscopy images (Fig. 1). This reconstruction shows that Blm10 binds in a defined orientation to both ends of the $20 \mathrm{~S}$ particle and contacts all the proteasome $\alpha$ subunits. Blm10 displays the solenoid folding observed in other HEAT-repeats (Fig. 2A) containing proteins and the axial gates on the $\alpha$ rings of the proteasome appear to be open (Fig. $2 \mathrm{~B} \&$ $\mathrm{C})$. These results indicate that the activation mechanism of the yeast $20 \mathrm{~S}$ proteasome by Blm 10 is similar to the one described for mammalian PA200 and that there is a functional and structural relationship between yeast B1m10 and mammalian PA200 proteins. Genetic analysis did not reveal a role for Blm10 in DNA repair, either alone or with Ecm29 (data not shown).

References

1.Voges, D., Zwickl, P., and Baumeister, W. (1999) Annu Rev Biochem 68, 1015-1068

2.Febres, D. E., Pramanik, A., Caton, M., Doherty, K., McKoy, J., Garcia, E., Alejo, W., and Moore, C. W. (2001) Cell Mol Biol (Noisy-le-grand) 47, 1149-1162

3.Ustrell, V., Hoffman, L., Pratt, G., and Rechsteiner, M. (2002) Embo J 21, 3516-3525.

4.Fehlker, M., Wendler, P., Lehmann, A., and Enenkel, C. (2003) EMBO Rep 4, 959-963

5.Schmidt, M., Haas, W., Crosas, B., Santamaria, P. G., Gygi, S. P., Walz, T., and Finley, D. (2005) Nat Struct Mol Biol 12, 294-303 


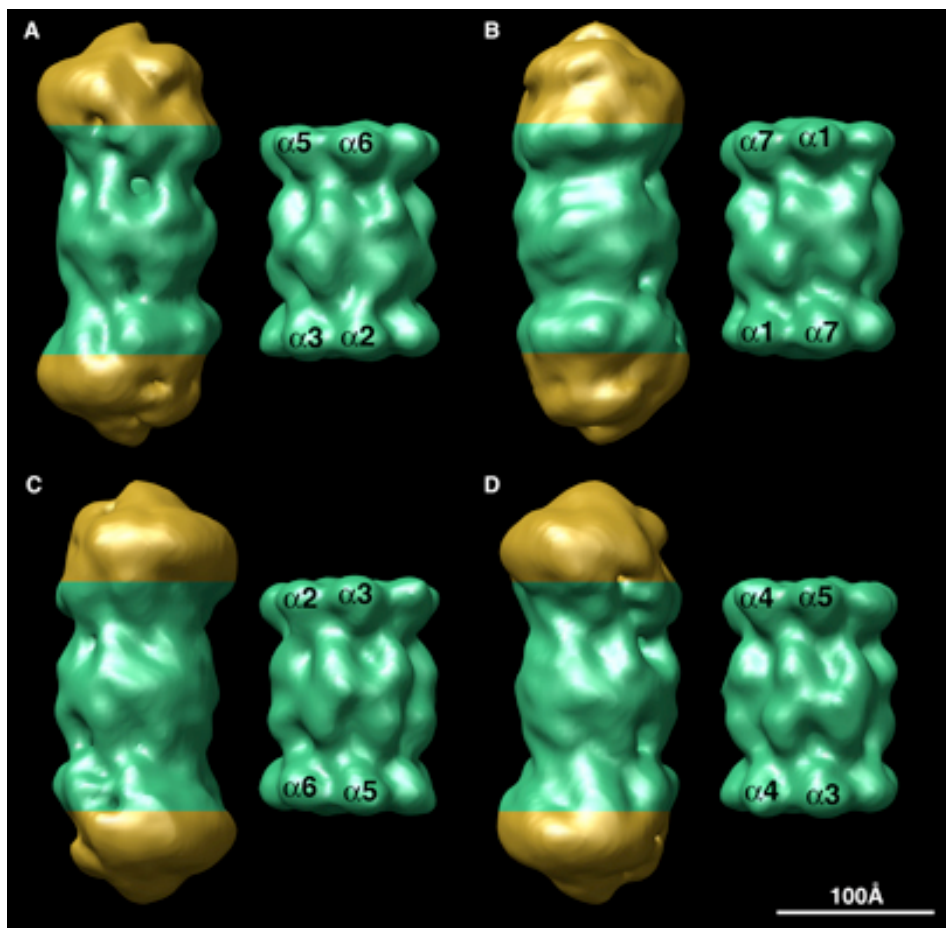

Fig. 1. Three-dimensional reconstruction of the Blm10-20S proteasome complex. Surface representation of the reconstruction of the $\mathrm{Blm} 10_{2}-20 \mathrm{~S}$ proteasome complex at $18 \AA$ resolution compared with the $\mathrm{X}$-ray structure of yeast 20S proteasome (PDB code:1RYP) limited at similar resolution. From (A) to (D) different side views of the complex are shown side by side with the analogous view of the $20 \mathrm{~S}$ proteasome. The contour used for the representation of the $\mathrm{B} 1 \mathrm{~m} 10_{2}$ 20S complex displays a molecular volume corresponding to a complex of 1.2 MDa. Blm10 are the non-symmetrical caps (colored in light brown) bound to the ends of the proteasome (colored in green). The two molecules of Blm10 contact the $\alpha$ rings at both ends of the proteasome in the same manner.

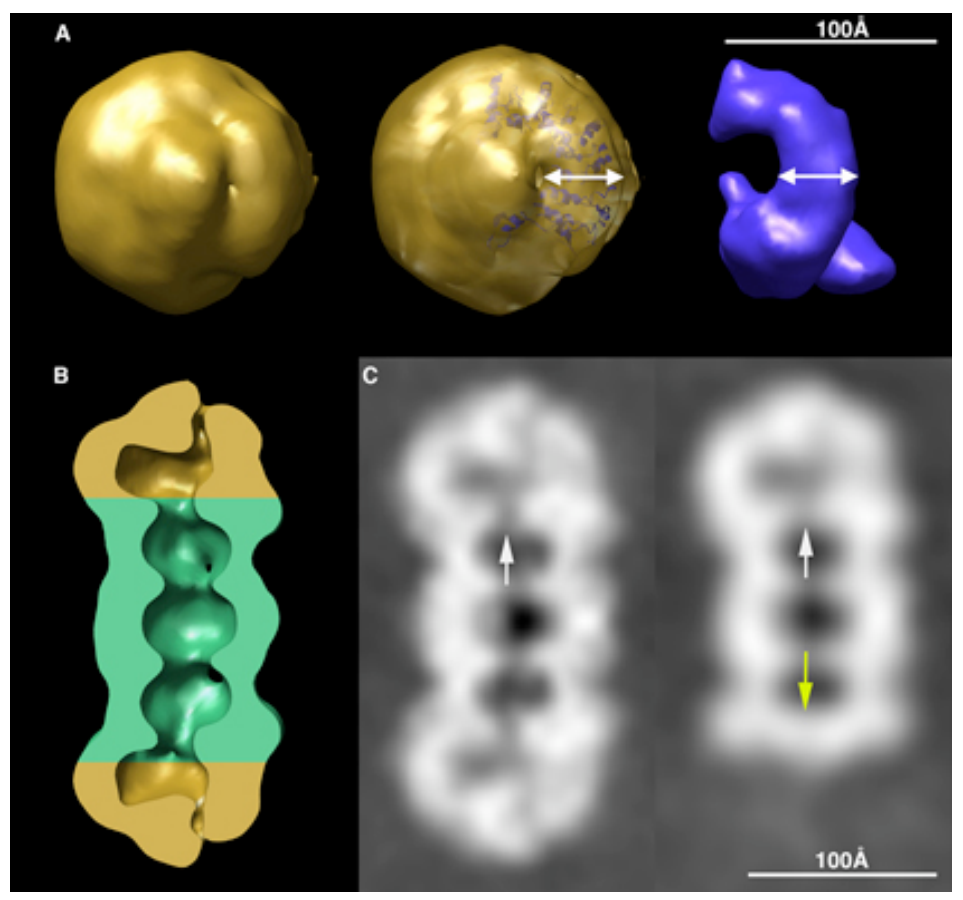

Fig. 2. Visualization of the solenoid folding of the Blm10 protein. Opening of the proteasomal axial channel upon Blm10 binding.

A, Surface representation of the axial view of the two Blm 10 caps in a Blm $10_{2}-20 \mathrm{~S}$ proteasome complex (left \& center panels) compared with the HEAT-repeat containing structure of karyopherin- $\beta 2$ protein (PDB code:1QBK) blurred to $18 \AA$ resolution (right panel). Double-headed arrows span the thickness of the solenoid in both proteins. Part of the atomic structure of karyopherin- $\beta 2$ (residues Tyr 3 to Leu 394) was docked into the solenoid path of the Blm10 cap (center panel). B, Sagital cut of the surface rendering representation of the $\mathrm{Blm} 10_{2}$ 20S proteasome complex. The light brown Blm10 caps at both ends of the 20S proteasome feature an internal cavity communicated with the digestion chamber of the proteasome by an opening of the axial channel. $\mathrm{C}$, Central section of the density maps of the Blm10 10 -20S (left panel) and Blm10-20S (right panel) complexes. Density in the center of the $\alpha$ rings in contact with Blm10 (white arrows) in both complexes is significantly lower than in the free $\alpha$ ring (yellow arrow) in the asymmetric complex. 